\title{
Dynamics of gene order rearrangements in mitochondrial genomes of Baikalian amphipods
}

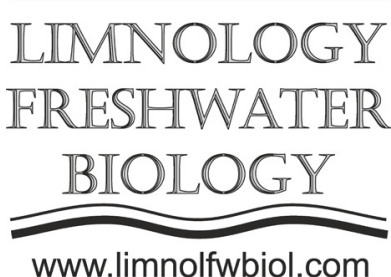

\author{
Sirotinina E.A.*, Romanova E.V., Sherbakov D.Yu. \\ Limnological Institute, Siberian Branch of the Russian Academy of Sciences, Ulan-Batorskaya Str., 3, Irkutsk, 664033, Russia
}

ABSTRACT. We describe peculiarities of shifts in mitochondrial gene order of different amphipod species in our study. Using phylogenetic inference and the methods of gene order rearrangement count we determine amphipod species with most highly rearranged mitochondrial genomes and attempt to explain the reasons for such variability.

Keywords: Lake Baikal, mitochondrial genomes, amphipods, gene rearrangement

\section{Introduction}

Gene order in the mitochondrial (mt) genomes of invertebrates is revealed to be different. The dynamics of such changes are usually studied by analysis of different organisms' lineages. It is noteworthy that the mitochondrial gene order maintained the same in some animal lineages for the long periods, whereas other lineages represent a considerable variation of this feature (Kilpert and Podsiadlowski, 2006). Amphipods can be a good model for studying mitochondrial gene order dynamics because of a relatively major number of species with sequenced $\mathrm{mt}$ genomes available (more than 100) and due to the variability of their mt gene order. Recent studies uncovered that endemic amphipods from Lake Baikal own rearranged $\mathrm{mt}$ genomes in comparison to the non-Baikalian species (Kilpert and Podsiadlowski, 2006; Romanova et al., 2016; Shen et al., 2017).

Amphipod species with currently sequenced $\mathrm{mt}$ genomes belong to different taxa and occupy different ecological niches, making it possible to suggest whether $\mathrm{mt}$ gene order rearrangements correlate with ecological features of species, their time of lineage divergence or life-history traits, etc. There was shown a positive correlation of the rate of gene rearrangements with the level of nucleotide substitutions in their protein-coding genes (Shao et al., 2003; Fourdrilis et al., 2018). We also recently demonstrated the significant increase in mutation rate in both lineages of Baikalian amphipods in comparison to species from gen. Gammarus (Romanova and Sherbakov, 2019).

There are two possible ways of the new gene orders emergence: one is about an erroneous duplication of the whole $\mathrm{mt}$ genome sequence during replication by "rolling ring" mechanism, associated with the DNA polymerase slippage on specific sites or repeats, and another, which involves a partial tandem duplication and random loss (TDRL) of a certain genome region (Xia et al., 2016). It is necessary to determine the number of mutational steps (permutations) from the ancestral gene order pattern to ones for the modern species to measure such gene rearrangements quantitatively. Using phylogenetic reconstructions, an ancestral gene pattern for any lineage is identified.

\section{Materials and Methods}

We defined an ancestral mt gene order pattern for all amphipods performing phylogenetic inference based on amino acid sequences of $13 \mathrm{mt}$ protein-coding genes. To estimate possible scenarios of gene order changing, including the minimum number of elementary gene permutations, we used software SORT2 and CREx (Bernt et al., 2007; Huang et al., 2010). In some cases we evaluated the changes manually by taking the number of displaced genes into account individually, and also counting the number of moved gene blocks grouped based on the proximity of their location.

\section{Results}

The analysis showed that Pancrustacean pattern (the general pattern for all crustaceans and insects) commonly used as a reference in mt gene rearrangements studies (Kilpert and Podsiadlowski, 2006; Krebes and Bastrop, 2012) differ from deduced Amphipoda pattern by the location of some tRNA genes. The Amphipoda pattern inferred in our study proved to be the same as one for gen. Gammarus species. The phylogenetic analysis revealed that this pattern was ancestral for both lineages of Baikalian amphipods. Thus the rearranged patterns of the majority of modern Baikalian amphipods

*Corresponding author.

E-mail address: haleo.inc@gmail.com (E.A. Sirotinina) 
were acquired during the evolutionary process in Lake Baikal. Remarkably, Baikalian amphipods from the second lineage (the one includes the majority of species which have very divergent morphological and ecological traits) possess gene order alteration affecting mostly tRNA gene positions. Whereas amphipod species from the first lineage, ones which are fewer in their species number and with a smooth body, inhabiting mainly in shallow water, have much more complicated alteration in their mt gene order, affecting the positions of the protein-coding and ribosomal genes (Romanova et al., 2016). We hypothesize that the fixation of highly rearranged mt genome becomes possible due to relaxed purifying selection in populations with low genetic diversity and low estimated effective population size. The shallow-water amphipod species in Lake Baikal could probably be more inclined to the striking decrease of their population and consequently genetic diversity due to environmental changes in the Lake Baikal.

\section{Acknowledgment}

The work was supported by the governmentally funded project 0345e2019e0004 (AAAA-A16-116122110060-9).

\section{References}

Bernt M., Merkle D., Ramsch K. et al. 2007. CREx: inferring genomic rearrangements based on common intervals. Bioinformatics 23: 2957-2958. DOI: 10.1093/ bioinformatics/btm 468

Fourdrilis S., de Frias Martins A.M., Backeljau T. 2018. Relation between mitochondrial DNA hyperdiversity, mutation rate and mitochondrial genome evolution in Melarhaphe neritoides (Gastropoda: Littorinidae) and other. Scientific Reports 8. DOI: 10.1038/s41598-018-36428-7
Huang Y.L., Huang C.C., Tang C.Y. et al. 2010. SoRT2: a tool for sorting genomes and reconstructing phylogenetic trees by reversals, generalized transpositions and translocations. Nucleic Acids Research 38: 221-227. DOI: 10.1093/nar/ gkq520

Kilpert F., Podsiadlowski L. 2006. The complete mitochondrial genome of the common sea slater, Ligia oceanica (Crustacea, Isopoda) bears a novel gene order and unusual control region features. BMC Genomics 7. DOI: 10.1186\%2F1471-2164-7-241

Krebes L., Bastrop R. 2012. The mitogenome of Gammarus duebeni (Crustacea Amphipoda): a new gene order and non-neutral sequence evolution of tandem repeats in the control region. Comparative Biochemistry and Physiology 7: 201-211. DOI: 10.1016/j.cbd.2012.02.004

Romanova E.V., Aleoshin V.V., Kamaltynov R.M. et al. 2016. Evolution of mitochondrial genomes in Baikalian amphipods. BMC Genomics 17. DOI: 10.1186/ s12864-016-3357-z

Romanova E.V., Sherbakov D.Y. 2019. Different rates of molecular evolution of mitochondrial genes in Baikalian and non-Baikalian amphipods. Limnology and Freshwater Biology 2: 339-344. DOI: $10.31951 / 2658-3518-2019-A-6$

Shao R., Dowton M., Murrell A. et al. 2003. Rates of gene rearrangement and nucleotide substitution are correlated in the mitochondrial genomes of insects. Molecular Biology and Evolution 20: 1612-1619. DOI: 10.1093/molbev/msg176

Shen Y., Kou Q., Zhong Z. et al. 2017. The first complete mitogenome of the South China deep-sea giant isopod Bathynomus sp. (Crustacea: Isopoda: Cirolanidae) allows insights into the early mitogenomic evolution of isopods. Ecology and Evolution 16: 1869-1881. DOI: 10.1002/ ece3.2737

Xia Y., Zheng Y., Murphy R.W. et al. 2016. Intraspecific rearrangement of mitochondrial genome suggests the prevalence of the tandem duplication-random loss (TDLR) mechanism in Quasipaa boulengeri. BMC Genomics 17. DOI: 10.1186/s12864-016-3309-7 